Conclusions GWAS and candidate gene studies discovered more than 300 lupus associated loci that explain the more than half of heritability in SLE, presumably leaving many SLE loci to be discovered in the future. Extensive studies have been performed in East Asians and Europeans with studies involving fewer subjects mean that we know less about SLE genetics in other populations. Many SLE associated variants are ancestry specific that have the potential to give distinction to clinical manifestations and disease prevalence when comparing ancestries. The great majority of SLE associated variants are located in non-coding regions; therefore, they more likely affect gene regulation, making this mechanism the key for understanding SLE genetics and pathogenesis. Surrogate candidate genes potentially affected by SLE associated polymorphisms appear to be mainly involved in immune processes and the regulation of transcription.

Acknowledgments Support is appreciated from US Department of Veterans Affairs Merit Award (I01 BX001834), and the National Institutes of Health (R01 AI24717 \& $\quad$ U01 AI130830).

\section{HIGH-THROUGHPUT IDENTIFICATION OF REGULATORY FUNCTIONAL SNPS ASSOCIATED WITH SYSTEMIC LUPUS ERYTHEMATOSUS}

${ }^{1}$ Qiang Wang, ${ }^{2}$ Marta Martínez Bonet, ${ }^{3}$ Matthew Weirauch, ${ }^{4}$ Peter A Nigrovic*. ${ }^{1}$ Boston Children's Hospital/Harvard Medical School, USA; ${ }^{2}$ Instituto de Investigación Sanitaria Gregorio Marañón, Spain; ${ }^{3}$ Cincinnati Children's Hospital Medical Center/University of Cincinnati, USA; ${ }^{4}$ Boston Children's Hospital/Brigham and Women's Hospital/Harvard Medical School, USA

\subsection{6/lupus-2021-lupus21century.86}

Background Systemic lupus erythematosus (SLE) is a disease involves the complex interplay of many genes, reflected in more than one hundred loci linked with disease risk by genome-wide association studies (GWAS). Decoding GWAS is therefore a promising strategy to identify novel drug targets in SLE. However, most of the identified disease-associated hits are noncoding single-nucleotide polymorphisms (SNPs), and cannot be distinguished from others that reside incidentally within risk loci. To address this longstanding challenge of finding the real regulatory functional SNPs (fSNPs) from among GWAS hits in SLE, we utilized an unbiased highthroughput screen method.

Methods From 5 GWAS for SLE (Gateva, Sandling et al. 2009, Bentham, Morris et al. 2015, Armstrong et al. 2015, Morris, Sheng et al. 2016, Langefeld, Ainsworth et al. 2017), 87 disease associated SNPs were chosen as lead SNPs and SNPs in linkage disequilibrium $(\mathrm{LD})\left(\mathrm{R}^{2}>0.8\right)$ with them are also included as the screening library. In total of 2176 SNPs were screened by three different high-throughput methods, SNP-seq (Li, Martinez-Bonet et al. 2018), H3K4me3 epigenetic modification (Trynka, Sandor et al. 2013), and Combined Annotation Dependent Depletion (CADD) (Rentzsch, Witten et al. 2018). Top candidates from the screening were further tested for regulatory function by electrophoretic mobility shift assay (EMSA) and luciferase reporter assay to define the final fSNPs candidates. Through bioinformatics binding motif prediction and mass spectrometry after oligo pulldown, transcriptional factors (TF) that might binds to the fSNPs were prioritized for validation by CHIP-qPCR, Western blot for oligo pulldown assay, and supershift. The association between selected fSNPs and the risk gene/associated gene was measured using genetic modified of the Daudi B cell line (CRISPRhomology directed repair). The association between the TF and the associated gene was measured by TF CRISPR knockout Daudi B cells.

Results Fifty-four candidate fSNPs from 2176 SNPs were found to be possible regulatory variants and tested for regulatory function. After EMSA, 9 SNPs showed allele-specific binding to proteins from both BL2 cells (B cell line) and PBMC nuclear extract. Six out of these 9 SNPs showed allelic differential gene expression in luciferase reporter assay in a B cell line (Daudi). After bioinformatics predictions as well as mass spectrometry for oligo pulldown assay, two fSNPs (rs2297550 and rs9907966) were found to be able to bind to transcriptional factor IKZF1 and YBX1 in B cells respectively. Specifically, IKZF1 prefers to bind to $G$ allele (risk allele) of rs2297550 and YBX1 prefers to bind to A allele (reference allele) of rs9907966. SNP rs2297550 was further analyzed for its association with the risk gene IKBKE and found out that the homozygous of risk $G$ allele is associated with lower IKBKE expression in $\mathrm{B}$ cells. Considering the role of IKBKE in preventing DNA damage induced cell death, the deficiency of IKBKE plausibly increases SLE risk. Through CRISPR knockout, we found out that the deficiency of TF IKZF1 leads to increased IKBKE expression. These data suggest a plausible mechanism which the risk allele of rs2297550 binds to IKZF1 to suppress the expression of IKBKE in B cells, therefore increase SLE risk.

Conclusions Our unbiased high-throughput screening for SLE GWAS hits followed by a step-wise validation leads to the identification of real functional regulatory fSNPs that are capable of binding to transcriptional factors and regulate gene expression, which establish a working model to bridge the gap between SLE GWAS and disease mechanism.

Acknowledgments This work was funded by a Target Identification in Lupus grant from the Lupus Research Alliance.

\section{THE INFLUENCE OF DIETARY RESISTANT STARCH CONTENT ON THE GUT MICROBIOTA OF PATIENTS WITH SYSTEMIC LUPUS ERYTHEMATOSUS AND ANTIPHOSPHOLIPID SYNDROME}

\begin{abstract}
1,2Iryna Kulyk, 'Marcia S Pereira, 'Sylvio Redanz, ${ }^{3}$ William E Ruff, ${ }^{4}$ Teri M Greiling, ${ }^{5}$ Carina Dehner, ${ }^{6}$ Odelya Pagovich, ${ }^{7}$ Daniel Zegarra Ruiz, ${ }^{8}$ Cassyanne L Aguiar, ${ }^{9}$ Doruk Erkan, ${ }^{1,3,10}$ Martin A Kriegel*. ${ }^{1}$ Department of Translational Rheumatology and Immunology, Institute for Musculoskeletal Medicine, University of Münster, Münster, Germany; ${ }^{2}$ Indiana University School of Medicine, Indianapolis, IN, USA; ${ }^{3}$ Department of Immunobiology, Yale University School of Medicine, New Haven, CT, USA; ${ }^{4}$ Department of Dermatology, Oregon Health and Science University, Portland, OR, USA; ${ }^{5}$ Department of Pathology, Washington University School of Medicine, St. Louis, MO, USA; ${ }^{6}$ Department of Genetic Medicine, Weill Cornell Medicine, New York, NY, USA; ${ }^{7}$ Memorial Sloan Kettering Cancer Center, New York, NY, USA; ${ }^{8}$ Department of Pediatric Rheumatology, Children's Hospital of The King's Daughters, Eastern Virginia Medical School, Norfolk, VA, USA; ${ }^{9}$ Barbara Volcker Center for Women and Rheumatic Diseases, Hospital for Special Surgery, Weill Cornell Medicine, New York, NY, USA; ${ }^{10}$ Section of Rheumatology and Clinical Immunology, Department of Internal Medicine D, University Hospital Münster, Münster, Germany
\end{abstract}

\subsection{6/lupus-2021-lupus21century.87}

Background Diets deficient in fibers are prevalent in modern societies and implicated in gut microbial dysbiosis contributing to the pathogenesis of chronic inflammatory disorders. ${ }^{1}$ A particular type of dietary fiber, resistant starch (RS) type 2, was shown to ameliorate disease in murine models of systemic lupus erythematosus (SLE). ${ }^{2}$ This effect was mediated by 
improvement of the gut barrier and growth inhibition of a translocating Lactobacillus strain. This Lactobacillus sp. was shown to drive lupus-related pathology in mice via the type I interferon pathway, and its genus was also enriched in a subset of SLE patients. Whether diet enriched in RS have similar effects in patients is unknown. We aimed to understand how dietary RS content influences gut microbial community structures in SLE and SLE-related antiphospholipid syndrome (APS) patients with well-defined microbiomes.,

Methods Stools and dietary information were collected from 12 SLE ( $n=28), 15$ APS $(n=44)$ patients and 20 control subjects $(n=48)$ for up to 3 visits $(0,4$ and 8 weeks $)$ as previously described $(3,4)$. Microbiota composition was defined by $16 \mathrm{~S}$ rRNA V4 region sequencing. The FDA reference list was used to calculate the RS content. Patients' diets were classified as low RS content if less than $2.5 \mathrm{~g}$ per day, medium RS if 2.5 to $15 \mathrm{~g}$, and high RS above $15 \mathrm{~g}$.

Results Lactobacillus spp. were significantly enriched in SLE patients $(p=0.002)$ compared to non-disease controls. APS patients showed a similar trend $(\mathrm{p}=0.06)$, but SLE patients displayed higher relative abundance compared to APS $(\mathrm{p}=0.011)$. No significant association was observed between low-to-medium RS content and Lactobacillus. High RS content was not achieved in routine diets of SLE and APS patients in these cohorts. However, medium RS was associated with an outgrowth of Bifidobacterium in SLE patients $(\mathrm{p}=0.016)$. Also, medium RS correlated in APS patients with a reduction of cardiolipin-synthesizing bacteria from the Coriobacteriaceae family $(\mathrm{p}=0.011)$ including Collinsella $(\mathrm{p}=0.009)$ and Slackia genera $(\mathrm{p}=0.033)$, previously linked to APS. ${ }^{5,6}$

Conclusions The content of RS in patients` regular diets has a distinct impact on the gut microbiota composition depending on the autoimmune disorder. Medium levels of dietary RS were associated in SLE with increased Bifidobacterium, shortchain fatty acid producing bacteria known to promote immune homeostasis, and with decreased cardiolipin-producing commensals in APS. It remains to be tested in an interventional trial if high RS content corrects the outgrowth of Lactobacillus in these patients, but moderate levels of RS may provide already beneficial effects on other taxa potentially involved in the pathogenesis of these disorders.

\section{REFERENCES}

1. Thorburn et al. 2014, Immunity 19, 833-842.

2. Zegarra-Ruiz et al. 2019, Cell Host Microbe 25, 113-127.

3. Greiling et al. 2018, Science Trans/ Med 10, 1-15.

4. Ruff et al. 2019, Cell Host Microbe 26, 1-14.

5. Aguiar et al. 2016, Arthritis Rheum 68 (suppl 10).

6. Bellochi et al. 2019, J Clin Med 8, 1291, 1-15.
Acknowledgments The work was supported by grants from the National Institutes of Health (NIH) (R01AI118855, T32AI07019), Arthritis National Research Foundation, Arthritis Foundation, Lupus Research Alliance, and Maren Foundation.

Trial Registrations ClinicalTrials.gov Identifiers: NCT02394964 (The Human Microbiome in Immune-Mediated Diseases) and NCT01787305 (Pilot Study of Gut Commensals in Antiphospholipid Syndrome)

\section{GENETICS: FLIPONS AND THE ROLE OF THE LEFT- HANDED Z-RNA CONFORMATION IN INTERFERONOPATHIES}

Alan Herbert*. InsidOuBio

\subsection{6/lupus-2021-lupus21 century.88}

Background In 1979, the first ever DNA crystal that was solved led to the surprising discovery of the left-hand Z-DNA conformation This structure forms from the right-handed classical B-DNA by flipping the bases over. The first protein shown to bind Z-DNA with high affinity was the doublestranded RNA editing protein ADAR1, but exactly why was uncertain for many years. A Proline to Alanine substitution at position 193 of ADAR1 (P193A) and an Asparagine to Serine substitution at position 173 (N173S), both in the Z $\alpha$ domain have been reported in families with Aicardi Goutières Syndrome type 6 , but also in the general population. Aicardi Goutières Syndrome causes severe neurological disease marked by dystonia with very few individuals surviving childhood. Disease is associated with a type I interferon signature.

Materials and Methods Structural and Genetic Analysis of ADAR1 biology

Results Evidence that P193A and N173S variants of the ZRNA binding domain, $Z \alpha$, of the RNA editing enzyme ADAR1 are causal for the Type I Interferonopathy Aicardi Goutières syndrome will be presented. The disease outcomes are driven by double-stranded RNAs derived from endogenous Alu inverted repeat retrotransposons. The Alu elements contain a Z-box with Z-RNA forming sequences that are normally bound by the $Z \alpha$ domain of ADAR1 to prevent activation of the double-stranded RNA sensor to MDA5 that drives the interferon response. The Alu elements mark hosttranscripts as self, preventing auto-reactivity.

Conclusions The regulation of type I Interferon responses by Z-RNA is the first time that a biological role for flipons has been conclusively confirmed. The P193A variant

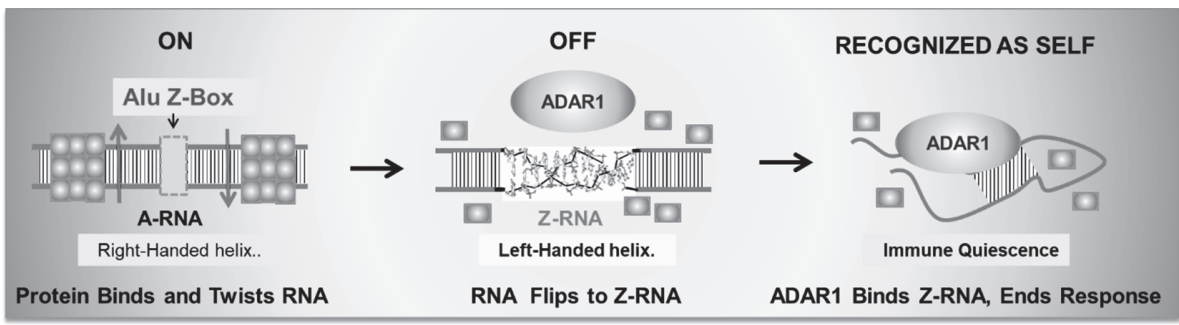

Abstract 1505 Figure 1 Double-stranded A-RNA (dsRNA) formed by Alu RNA elements is twisted and shortened as the helicase MDA5 engages it. If the right-handed dsRNA (helical length $=2.46 \mathrm{~nm}$ ) contains a Z-Box, then the tension generated is relieved by flipping the sequence to the longer left-handed Z-RNA conformation (helical length $=4.56 \mathrm{~nm}$ ). The flip causes the immune proteins to fall off the RNA. The Z-RNA formed engages the editing enzyme ADAR1. ADAR1 modifies dsRNA, producing single-stranded RNA that does not cause immune activation. The Z-Box sequences are called flipons. 